\title{
Understanding the Motions of Tools and Vehicles
}

\author{
Zoran Duric ${ }^{1,2} \quad$ Ehud Rivlin ${ }^{2,3}$ \\ ${ }^{1}$ Computer Science Department \\ George Mason University \\ Fairfax, VA 22030-4444 \\ ${ }^{3}$ Department of Computer Science \\ ${ }^{2}$ Center for Automation Research \\ University of Maryland \\ College Park, MD 20742-3275 \\ Technion - Israel Institute of Technology
Haifa, Israel 32000
}

\begin{abstract}
Many types of common objects, such as tools and vehicles, usually move in simple ways when they are wielded or driven: The natural axes of the object tend to remain aligned with the local trihedron defined by the object's trajectory. Based on this observation we use a model called Frenet-Serret motion which corresponds to the motion of a moving trihedron along $a$ space curve. Knowing how the Frenet-Serret frame is changing relative to the observer gives us essential information for understanding the object's motion. This is illustrated here for four examples, involving tools ( $a$ wrench and a saw) and vehicles (an accelerating van, a turning taxi).
\end{abstract}

\section{Introduction}

An object moves because it is self-propelled (e.g., a vehicle) or because it is wielded, pushed, or pulled (or thrown ${ }^{1}$ ) by an agent (e.g., a tool). Motion that efficiently performs a locomotional or mechanical function requires efficient energy transfer from the vehicle's engine or the agent's arm to the object, in order to efficiently overcome the constraints imposed by the environment in which the motion takes place (air resistance, friction, etc.). Assuming that an object has natural axes (e.g. the long axis of a stick), efficient force transfer requires simple relationships between the natural axes of the object and the motion trajectory. These relationships insure that the object can perform its function efficiently.

The most general model of object motion is unrestricted rigid motion. This type of motion is not common in everyday life. Usually objects are supported, and motion takes place when an object is in contact with a surface, another object, or an agent. In these cases (tool acting on a recipient object; ground vehicle) the motion becomes interestingly constrained.

\footnotetext{
${ }^{1}$ We assume in this paper that the propulsive force is applied to the object continuously, unlike the case of a projectile where it is applied only initially. We will not discuss projectiles further here.
}

In our work we consider the relationship between this constrained motion and the object's geometry. To analyze this relationship we use two frames: the object frame and the frame of the motion trajectory. "Efficient" motion calls for a simple relationship between the object frame and the motion frame, and this relationship remains constant during the motion. Based on this observation we use a model called Frenet-Serret motion which corresponds to the motion of a moving trihedron along a space curve [8]. The parameters of the motion are given by the curvature and torsion of the space curve along which the object moves.

In practice the simple nature of the environment in which the object moves provides further constraints. A ground vehicle is moving on relatively flat terrain, and a tool is often acting on a planar surface. The motion is mostly planar (though the plane might rotate slightly through the motion). Over a long time period the motion is Frenet-Serret and over a short time period the motion is approximately planar and often approximately translational.

We use the relationship between the object frame and the motion frame to analyze image sequences. Given a sequence of images of the moving object, our analysis enables us to output the motion and trajectory parameters of the object. Knowing how the Frenet-Serret frame is changing relative to the observer gives us essential information for understanding the object's motion. Our analysis can also handle constraints on the motion. For example, the parameters of the object's trajectory depend on its speed, mass, size, and on the medium through which it moves. These factors impose bounds on the curvature and torsion of the trajectory.

In this paper we approach object motion understanding through analysis of long image sequences. A key question in this context is how to relate shortsequence motion estimation to long-sequence motion estimation. Using the Frenet-Serret frame provides us with an ability to understand motion over a long time period. We can derive the motion parameters from the parameters of the trajectory and obtain motion 
descriptions suitable for long sequence analysis. Using these tools we can show, for example, that rotation becomes significant only in long sequences, and that in a short sequence translation is usually dominant. We show that using simplified scene and imaging models we can get adequate local estimates (short sequence, 2-4 frames) by analyzing the images, and by observing these estimates over a long sequence we can accumulate them to describe the object's trajectory. Analysis of the trajectory parameters provides us with tools for understanding long-term object motion.

\section{Related Work}

Understanding object motion is based on extracting the object's motion parameters from an image sequence. Broida and Chellappa [1] proposed a framework for motion estimation of a vehicle using Kalman filtering. Weng et al. [18] assumed an object that possesses an axis of symmetry, and a constant angular momentum model which constrained the motion over a local frame subsequence to be a superposition of precession and translation. The trajectory of the center of rotation can be approximated by a vector polynomial. Changing the parameters of the model with time allows adaptation to long-term changes in the motion characteristics. Their work was based on correspondence; at least eight pairs of corresponding points were needed.

Accumulating the information obtained from the motion analysis of the sequence to achieve an estimate of the moving object's trajectory is another step toward understanding object motion. (A good survey of motion-based recognition was compiled by $\mathrm{Ce}$ dras and Shah [5].) Bruckstein et al. [2,3] assumed a known object model (a rigid rod or disk) and tried to recover the object's trajectory and rotation. They showed that five images are enough to recover the motion of a rod or a disk in accordance with physical laws. Techniques from algebraic geometry were used to establish the existence of solutions to the resulting polynomial equations.

Engel and Rubin [11] (and similarly Gould and Shah [13] used motion characteristics obtained by tracking representative points on an object to identify important events corresponding to changes in direction, speed and acceleration in the object's motion.

Work has also been done on model-based tracking (e.g., [15]) and on higher-level descriptions of object trajectories in terms of such concepts as stopping/starting, object interactions, and motion verbs $[4,14,16]$. These levels of object motion description will not be treated in this paper.

In [6] Duric et al. tried to determine the function of an object from its motion. Given a sequence of images of a known object performing some function, they attempted to determire what that function was. They showed that the motion of an object, when com- bined with information about the object and its uses, provides strong constraints on the possible function being performed. Their flow-based analysis treated relatively short sequences.

In this paper a model for object trajectory analysis is used, and a constant relationship between the object frame and the motion frame is established.

\section{Motion Models}

\subsection{Rigid Body Motion}

To facilitate the derivation of the motion equations of a rigid body $\mathcal{B}$ we use two rectangular coordinate frames, one $(O x y z)$ fixed in space, the other $\left(C x_{1} y_{1} z_{1}\right)$ fixed in the body and moving with it. The position of the moving frame at any instant is given by the position $\vec{d}_{c}=\left(\begin{array}{lll}X_{c} & Y_{c} & Z_{c}\end{array}\right)^{T}$ of the origin $C$, and by the nine direction cosines of the axes of the moving frame with respect to the fixed frame. For a given position $\vec{p}$ of $P$ in $C x_{1} y_{1} z_{1}$ we have the position $\vec{r}_{p}$ of $P$ in $O x y z$

$$
\vec{r}_{p}=R \vec{p}+\vec{d}_{c}
$$

where $R$ is the matrix of the direction cosines (the frames are taken as right-handed so that $\operatorname{det} R=1$ ). The velocity $\overrightarrow{\vec{r}}_{p}$ of $P$ in $O x y z$ is given by

$$
\dot{\vec{r}}_{p}=\vec{\omega} \times\left(\vec{r}_{p}-\vec{d}_{c}\right)+\dot{\vec{d}}_{c}
$$

where $\dot{\vec{d}}_{c}$ is the translational velocity vector and $\vec{\omega}=$ $\left(\begin{array}{lll}\omega_{x} & \omega_{y} & \omega_{z}\end{array}\right)^{T}$ is the rotational velocity vector.

\subsection{Motion along a Smooth Curve}

Consider a moving frame $C x_{1} y_{1} z_{1}$ (fixed in a rigid body $\mathcal{B}$ ), which moves with $C$ along a space curve $\Gamma$ while rotating so that the $C x_{1}$ and $C y_{1}$ axes coincide with, respectively, the tangent and principal normal of $\Gamma$. This means that as $C$ moves along $\Gamma$ the $C x_{1} y_{1} z_{1}$ frame coincides with the Frenet-Serret trihedron at $C: C t n b$. This trihedron consists of the tangent $\overrightarrow{\mathbf{t}}$, the principal normal $\overrightarrow{\mathbf{n}}$, and the binormal $\vec{b}$, which are mutually orthogonal. The geometry of this motion is completely defined by $\Gamma$.

Let $\vec{d}_{\gamma}(s)$ denote the position of $C$, in the fixed coordinate frame $O x y z$, when it has moved along $\Gamma$ through a total arc length of $s$. For any position $\vec{p}$ of a point $\mathrm{P}$ on $\mathcal{B}$ in $C t n b$, the position $\vec{r}_{p}$ in $O x y z$ is given by (1). If $\overrightarrow{\mathbf{t}}=\left(\begin{array}{lll}t_{1} & t_{2} & t_{3}\end{array}\right)^{T}, \overrightarrow{\mathbf{n}}=\left(\begin{array}{lll}n_{1} & n_{2} & n_{3}\end{array}\right)^{T}$ and $\overrightarrow{\mathbf{b}}=\left(\begin{array}{lll}b_{1} & b_{2} & b_{3}\end{array}\right)^{T}$ are the unit vectors along $C t, C n$ and $C b$, differential geometry gives us

$$
\overrightarrow{\mathbf{t}}=\vec{d}_{\gamma}^{\prime}, \quad \overrightarrow{\mathbf{n}}=\kappa^{-1} \vec{d}_{\gamma}^{\prime \prime}, \quad \overrightarrow{\mathbf{b}}=\overrightarrow{\mathbf{t}} \times \overrightarrow{\mathbf{n}},
$$


where $\kappa$ is the curvature of $\Gamma$. Also, the columns of $R$ are the vectors $\overrightarrow{\mathbf{t}}, \overrightarrow{\mathbf{n}}$, and $\overrightarrow{\mathbf{b}}$. We have the Frenet-Serret formulas $[17]$

$$
\overrightarrow{\mathbf{t}}^{\prime}=\kappa \overrightarrow{\mathbf{n}}, \quad \overrightarrow{\mathbf{n}}^{\prime}=-\kappa \overrightarrow{\mathbf{t}}+\tau \overrightarrow{\mathbf{b}}, \quad \overrightarrow{\mathbf{b}}^{\prime}=-\tau \overrightarrow{\mathbf{n}}
$$

where $\tau$ is the torsion of $\Gamma$. From (4) and the definition of $R,(2)$ can be written as

$$
\vec{r}_{p}^{\prime}=\vec{\omega}_{d} \times\left(\vec{r}_{p}-\vec{d}_{\gamma}\right)+\overrightarrow{\mathbf{t}}
$$

where the Darboux vector $\vec{\omega}_{d}=\tau \overrightarrow{\mathbf{t}}+\kappa \overrightarrow{\mathrm{b}}$ is the rotational velocity vector and the unit tangent $\overrightarrow{\mathbf{t}}$ of $\Gamma$ is the translational velocity vector; the motion parameter is the arc length $s$. If, instead of using arc length as a motion parameter, time $t$ is used, the rotational velocity $\vec{\omega}_{d}$ and translational velocity $\overrightarrow{\mathrm{t}}$ are scaled by the speed $v=d s / d t$ of the point $C$. In the special case where $\Gamma$ is a plane curve we have $\tau=0$ ( $\Gamma$ is torsionless), and thus $\vec{\omega}_{d}=\kappa \overrightarrow{\mathbf{b}}$. We then have from (5)

$$
\dot{\vec{r}}_{p}=v \kappa \overrightarrow{\mathbf{b}} \times\left(\vec{r}_{p}-\vec{d}_{\gamma}\right)+v \overrightarrow{\mathbf{t}}
$$

\subsection{Simple Motions of Objects}

Objects move in reaction to forces which are being applied to them. When the forces acting on an object are added, the resultant force $\vec{F}$ determines the direction of motion and the moments of the forces (or the torques) determine the rotation of the object. If the force $\vec{F}$ is applied to the object $\mathcal{B}$ at the point $P$, the moment $\vec{M}$ is given by $\vec{M}=\vec{r}_{p} \times \vec{F}$ where $\vec{r}_{p}$ is the position of $P$ relative to a point $C . \vec{M}$ has the same direction as the axis of the rotation of $\mathcal{B}$ that results from applying $\vec{F}$

The engine of a vehicle needs to apply force to the vehicle in order to move it from one position to another. If the path is prespecified (as in the case of a ground vehicle on a road), effective application of the force requires that the angle between the instantaneous directions of the force and the directions of the path elements be small. The force differential generates torques which help turn the vehicle around the axis of rotation normal to the (osculating) plane of the path. During a turn, the wheels rotate with different speeds; the greater the distance between the wheels the larger their difference in speed. In order to minimize this difference the distance between the wheels needs to be small. Also, when forces are applied to the wheels the resulting torques are larger when the vehicle is moving along a short axis; but these torques need to be as small as possible to improve the handling of and minimize stresses on the vehicle. Because of all these factors the principal axis of inertia of the vehicle should be tangent to the path of the vehicle. It should be pointed out that [7] the translational velocity at any point on a ground vehicle is typically orders of magnitude larger than its rotational velocity (around the vehicle's center of mass). The rotational velocity becomes significant only when the vehicle is observed over a significant period of time (typically several frames).

In the case of a moving tool the force is used not only to move the tool, but to act on a recipient object. Therefore, the required force depends on the task. For example, sawing involves continuously exerting a force perpendicular to the path of the saw; tightening with a wrench involves continuously exerting torque around the axis of rotation. (Note that the force may not be applied to the recipient object continuousty; for example, when we swing a hammer, the force is applied only when the head of the hammer hits the object.) Developing a general theory of tool motion is a subject of our continuing research.

\section{Tool Motion}

We assume that the tool is (approximately) planar and that its velocity is composed of a translational velocity in the plane of the tool and a rotational velocity around an axis orthogonal to the plane of the tool. For the purpose of estimating object motion from images we rewrite equation (2) in the following way:

$$
\dot{\vec{r}}_{p}=\vec{\omega} \times\left(\vec{r}_{p}-\vec{d}_{c}\right)+\dot{\vec{d}}_{c}=\vec{\omega} \times \vec{r}_{p}+\vec{T}
$$

where $\vec{T}=\dot{\vec{d}}_{c}-\vec{\omega} \times \vec{d}_{c} \equiv(U V W)^{T}$ is the translational velocity expressed in the fixed (camera) coordinate frame Oxyz. We will later show how the translational velocity $\dot{\vec{d}}_{c}$ can be recovered from $\vec{T}$.

\subsection{The Image Motion Field of a Wielded Tool}

Let the normal to the plane be $\vec{N}=\left(\begin{array}{lll}N_{x} & N_{y} & N_{z}\end{array}\right)^{T}$ and let the plane intersect the $z$-axis at the point $\left(0,0, Z_{0}\right)$ of the $O x y z$ coordinate frame. If we assume a nondegenerate view (i.e., $N_{z}>0$ ) for points on the plane we can define $p=N_{x} N_{z}^{-1}$ and $q=N_{y} N_{z}^{-1}$. From our assumption about rotational velocity it follows that we have $\vec{\omega}=\left(\begin{array}{lll}p \omega_{z} & q \omega_{z} & \omega_{z}\end{array}\right)$ for some $\omega_{z}$. Also, since we have assumed that the translation is in the plane of the tool we have $\vec{N} \cdot \vec{T}=0$, or equivalently

$$
W=-U p-V q .
$$

We define scaled components of the translational velocity $Z_{0}^{-1} U \equiv U_{0}$ and $Z_{0}^{-1} V \equiv V_{0}$ and $Z_{0}^{-1} W \equiv W_{0}$.

Given the point $\vec{r}=x \vec{\imath}+y \vec{\jmath}$ and the normal direction $n_{x} \vec{\imath}+n_{y} \vec{j}$, the normal motion field $\dot{\vec{r}}_{n} \cdot \vec{n}=$ $n_{x} \dot{x}+n_{y} \dot{y}$ is given by

$$
\dot{\vec{r}}_{n} \cdot \vec{n}=U_{0} \varphi_{1}+V_{0} \varphi_{2}+\omega_{z} \varphi_{3}
$$

where the $\varphi$ s are nonlinear functions of $p, q, \vec{r}$, and $\vec{n}$ given by

$$
\varphi_{1}=(f+p x+q y)\left[n_{x}+\left(x n_{x}+y n_{y}\right) p f^{-1}\right],
$$




$$
\begin{aligned}
\varphi_{2}= & (f+p x+q y)\left[n_{y}+\left(x n_{x}+y n_{y}\right) q f^{-1}\right], \\
\varphi_{3}= & n_{x}\left(-y+q f-p x y f^{-1}+q x^{2} f^{-1}\right) \\
& +n_{y}\left(x-p f+q x y f^{-1}-p y^{2} f^{-1}\right) .
\end{aligned}
$$

A detailed derivation of (9) can be found in [9] and [10].

In (9) $\vec{r}$ and $\vec{n}$ are observable from images, while the 5-tuple $\left(p, q, U_{0}, V_{0}, \omega_{z}\right)$ is not directly observable. To estimate this 5-tuple we need estimates of $\dot{\vec{r}}_{n} \cdot \vec{n}$ at five or more image points.

\subsection{Estimating Tool Motion from Normal Flow}

If we use the spatial image gradient as the normal direction $\vec{n}_{r} \equiv \nabla I /\|\nabla I\|=n_{x} \vec{\imath}+n_{y} \vec{\jmath}$ and $\dot{\vec{r}}_{n} \approx \vec{u}_{n}$ we can obtain an approximate equation corresponding to (9) by replacing the left hand side of (9) by normal flow $-I_{t} /\|\nabla I\|$. This equation involves the eight unknown elements of $\mathbf{c}$. For each point $\left(x_{i}, y_{i}\right)$, $i=1, \ldots, m$ of the image at which $\left\|\nabla I\left(x_{i}, y_{i}, t\right)\right\|$ is large we can write one such equation. If we have $m$ such points, where $m \gg 5$, we have an overdetermined system of equations

$$
\Phi(p, q) \cdot\left(U_{0} V_{0,} \omega_{z}\right)^{T} \approx \mathbf{b}
$$

where the $m \times 3$ matrix function $\Phi$ is given by

$$
\Phi(p, q)=\left[\varphi_{1}(p, q) ; \varphi_{2}(p, q) ; \varphi_{\mathbf{3}}(p, q)\right]
$$

(i.e., its columns are $m$-vectors that correspond to values of $\phi$ at points $\left.\left(x_{i}, y_{i}\right)\right)$, and the elements of the $m$-vector $\mathrm{b}$ are $-\left(\partial I\left(x_{i}, y_{i}, t\right) / \partial t\right) /\left\|\nabla I\left(x_{i}, y_{i}, t\right)\right\|$.

We seek the solution of the system (10) for which $\left\|\mathbf{b}-\Phi \cdot\left(\begin{array}{lll}U_{0} & V_{0} & \omega_{z}\end{array}\right)^{T}\right\|$ is minimal - i.e., we are seeking the solution of $(10)$ in the least squares sense. This is a separable nonlinear least squares problem; a good stable solution and an algorithm were given in [12]. It was shown that the problem is equivalent to minimizing

$$
r(p, q)=\left\|\mathbf{b}-\Phi(p, q) \Phi^{+}(p, q) \mathbf{b}\right\|,
$$

where $\Phi^{+}$is the generalized inverse of $\Phi . r(p, q)$ is first minimized to obtain optimal values $\hat{p}$ and $\hat{q}$ of $p$ and $q$ respectively; these values are then used to obtain $\Phi(\hat{p}, \hat{q})$. The linear least squares method is then used to minimize $\left\|\mathbf{b}-\Phi(\hat{p}, \hat{q}) \cdot\left(\begin{array}{lll}U_{0} & V_{0} & \omega_{z}\end{array}\right)^{T}\right\|$ and obtain optimal values of the motion parameters $U_{0}, V_{0}$, and $\omega_{z}$. After estimating $p, q, U_{0}, V_{0}$, and $\omega_{z}$ we (8) to obtain $W_{0}$. Finally, we obtain $\vec{N}=\left(\begin{array}{lll}p & q & 1\end{array}\right)^{T}\left(1+p^{2}+\right.$ $\left.q^{2}\right)^{-\frac{1}{2}}$ and $\|\vec{\omega}\|=\sqrt{\omega_{z}^{2}+p^{2} \omega_{z}^{2}+q^{2} \omega_{z}^{2}}$.

We have estimated the translational velocity $\vec{T}$ and the rotational velocity $\vec{\omega}$ in the camera coordinate system $O x y z$. We are interested in the translational and the rotational velocity expressed in the FrenetSerret frame Otnb. By comparing (2), (6) and (7) we obtain

$$
\vec{\omega}=v \kappa \overrightarrow{\mathrm{b}}, \quad \overrightarrow{\mathrm{b}}=\vec{N} \operatorname{sgn} \omega_{z}, \quad v \kappa=\|\vec{\omega}\|
$$

where sgn stands for the 'sign of' function. Let $\vec{d}_{\gamma}=$ $\left(\begin{array}{llll}X_{c} & Y_{c} & Z_{c}\end{array}\right)^{T}$ be the position of $C$ and let $\left(x_{c}, y_{c}\right)$ be the image of $C$ (either the tip or the center of mass of the tool). We have

$$
\frac{v \overrightarrow{\mathbf{t}}}{Z_{0}}=\left(\begin{array}{lll}
U_{0} & V_{0} & W_{0}
\end{array}\right)^{T}+\frac{\vec{\omega} \times\left(x_{c} y_{c} f\right)^{T}}{f+p x_{c}+q y_{c}} .
$$

From (13) we obtain the unit vector in the tangent direction $\overrightarrow{\mathbf{t}}$ by normalizing $v \overrightarrow{\mathbf{t}} / Z_{0}$. Finally, we obtain the unit vector in the normal direction using

$$
\overrightarrow{\mathbf{n}}=\overrightarrow{\mathbf{b}} \times \overrightarrow{\mathbf{t}} .
$$

Equations (12), (13) and (14) define the Frenet-Serret frame Otnb expressed in the camera coordinate system. Equation (12) gives us the curvature $\kappa$ up to an unknown factor $v$ (linear velocity). We conclude that the Frenet-Serret motion can be recovered up to the speed $v$; note that the translational velocity $v \overrightarrow{\mathbf{t}} / Z_{0}$ does not help here because of the unknown depth $Z_{0}$.

Finally, we need to recover the orientation of the tool coordinate frame (its long and short axes) in the $O t n b$ frame. We find the long and the short axes of the tool as the principal axes of the set of tool points. The long axis $l$ of the tool and the origin $O$ of the fixed (camera) coordinate frame Oxyz define a plane $\Pi_{l}$. Since the image $l^{\prime}$ of $l$ lies in this plane we can find $P i_{l}$ using $l^{\prime}$ in place of $l$. Because we have assumed a nondegenerate view we have two cases: (i) if the tangent vector $\overrightarrow{\mathbf{t}}$ lies in $\Pi_{l}$ the motion is along $l$; (ii) if the normal vector $\overrightarrow{\mathbf{n}}$ lies in $\Pi_{l}$ the motion is orthogonal to $l$.

We check if the vector lies in the plane $\Pi_{\gamma}$ using the following simple algorithm. Let $\vec{p}_{1}=\left(\begin{array}{lll}x_{1} & y_{1} & f\end{array}\right)^{T}$ and $\vec{p}_{2}=\left(\begin{array}{lll}x_{2} & y_{2} & f\end{array}\right)^{T}$ be the positions of two endpoints on the image $l^{\prime}$ of $l$. The normal $\vec{N}_{\Pi}$ of the plane $\Pi_{l}$ is given by

$$
\vec{N}_{\Pi}=\vec{p}_{1} \times \vec{p}_{2} .
$$

If the vector $\overrightarrow{\mathrm{t}}$ lies in the plane $\Pi_{l}$ we have $\vec{N}_{\Pi} \times \overrightarrow{\mathrm{t}} \approx 0$. So to find out the relative orientation of the tool frame and the Otnb frame we only need to find which one of the inner products $\left|\vec{N}_{\Pi} \cdot \overrightarrow{\mathbf{t}}\right|$ and $\left|\vec{N}_{\Pi} \cdot \overrightarrow{\mathbf{n}}\right|$ is smaller. (Note that while one of the vectors $\overrightarrow{\mathbf{t}}$ and $\overrightarrow{\mathbf{n}}$ lies in the plane $\Pi_{l}$ the other vector is not always orthogonal to $\left.\Pi_{l}.\right)$

\section{Vehicle Motion}

We assume that the motion of the vehicle is planar and that it has a small rotational velocity around the axis orthogonal to the plane of motion. The translational velocity is dominant and at any time $t$ the motion can be approximated by pure translational motion. 


\subsection{The Image Motion Field of a Moving Vehicle}

Given the point $\vec{r}=x \vec{\imath}+y \vec{\jmath}$ and the normal direction $n_{x} \vec{\imath}+n_{y} \vec{\jmath}$, the (approximate) normal motion field for points on a vehicle under weak perspective $\dot{\vec{r}}_{n} \cdot \vec{n}=n_{x} \dot{x}+n_{y} \dot{y}$ is given by

$$
\dot{\vec{r}}_{n} \cdot \vec{n}=n_{x} f U Z_{c}^{-1}+n_{y} f V Z_{c}^{-1}-\left(n_{x} x+n_{y} y\right) W Z_{c}^{-1}
$$

Let

$$
\mathbf{a}=\left(\begin{array}{c}
n_{x} f \\
n_{y} f \\
-n_{x} x-n_{y} y
\end{array}\right), \quad \mathbf{c}=\left(\begin{array}{c}
U Z_{c}^{-1} \\
V Z_{c}^{-1} \\
W Z_{c}^{-1}
\end{array}\right)
$$

Using these expression we can write $(15)$ as $\dot{\vec{r}}_{n} \cdot \vec{n}=$ $\mathbf{a}^{T} \mathbf{c}$. The column vector $\mathbf{a}$ is formed of observable quantities only, while each element of the column vector c contains quantities which are not directly observable from the images. To estimate c, the scaled translational velocity $Z_{c}^{-1} \vec{T}=Z_{c}^{-1}\left(\begin{array}{lll}U & V\end{array}\right)^{T}$, we need estimates of $\dot{\vec{r}}_{n} \cdot \vec{n}$ at three or more image points.

\subsection{Estimating Vehicle Motion from Nor- mal Flow}

Similarly to our method in Section 4.2 we use linear least squares to estimate parameter vector $\mathbf{c}$ from the normal flow.

In the case of a moving vehicle the parameters of interest are the vehicle's trajectory and its rate of approach. The rate of approach

$$
\nu=\frac{W}{Z_{c}}
$$

(measured in $\mathrm{sec}^{-1}$ ) is equivalent to the inverse of the time to collision and corresponds to the rate with which an object is approaching the camera (or receding from it). The rate $\nu=0.1 / \mathrm{sec}$ means that every second the object travels 0.1 of the distance between the observer and its current position. A negative rate of approach means that the object is going away from the camera.

The direction of motion $\mathrm{c} \equiv \vec{T} / Z_{c}$ gives us the tangent vector $\overrightarrow{\mathbf{t}}=\mathbf{c} /\|\mathbf{c}\|$ of the Frenet-Serret frame. If the direction of motion changes over time we can use the Frenet-Serret formulas (4) to recover the (scaled) curvature $v \kappa$ of the trajectory. Given the tangent direction $\overrightarrow{\mathbf{t}}_{0}$ at time $t$ and the tangent direction $\overrightarrow{\mathbf{t}}_{1}$ at time $t+\Delta t$ we have

$$
\overrightarrow{\mathbf{n}}_{0}=v \kappa \overrightarrow{\mathbf{n}} \approx \frac{\overrightarrow{\mathbf{t}}_{1}-\overrightarrow{\mathbf{t}}_{0}}{\Delta t}
$$

The unit vector in the direction $\overrightarrow{\mathbf{n}}_{0}$ at time $t$ is the normal vector of the Otnb frame and the scaled curvature is given by $v \kappa=\left\|\overrightarrow{\mathbf{n}}_{0}\right\|$. Finally, we obtain

$$
\overrightarrow{\mathbf{b}}=\overrightarrow{\mathbf{t}} \times \overrightarrow{\mathbf{n}} \text {. }
$$

Equations (16) and (17) give us the normal $\overrightarrow{\mathbf{b}}$ to the plane of motion and the rotational velocity of turning (yaw) $\vec{\omega}=v \kappa \overrightarrow{\mathrm{b}}$.
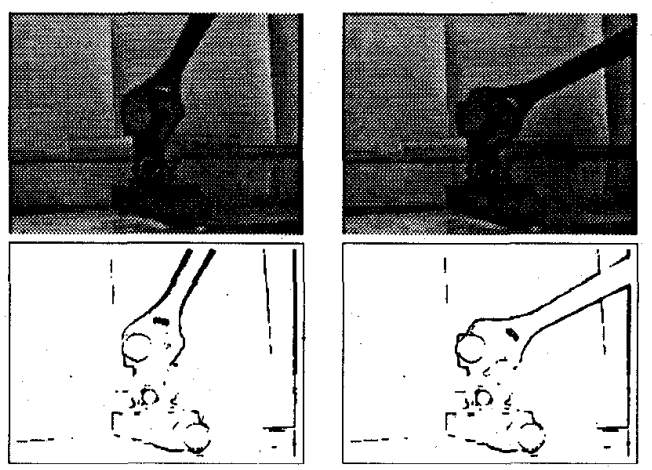

Figure 1: An experiment using a wrench: frames 30 and 100. Top images: the input images. Bottom images: results of flow computation.

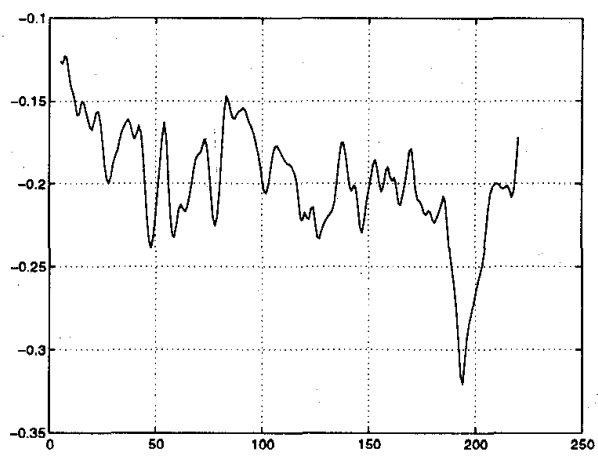

Figure 2: Results of experiments on the wrench sequence: the graph shows rotational velocity in radians/sec.

\section{Experiments}

In the following section we show two examples for each of the domains we have discussed: tools and vehicles. As was mentioned before, tools usually operate by planar motion, advancing along a line (drill) or moving in a plane (sawing). In our examples we show two types of motion: rotation with negligible translation, and relatively small rotation and dominant translation. In Section 6.1 we will analyze saw and wrench examples.

A ground vehicle's motion usually takes place on terrain that has a small slope and on a road with a limited rate of turn. This results in small values of pitch 


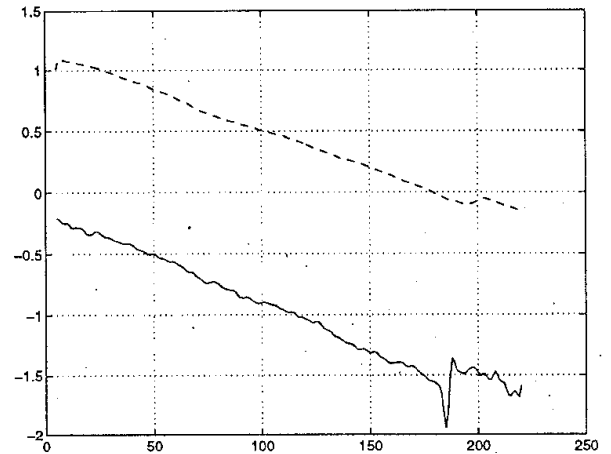

Figure 3: Results of experiments on the wrench sequence. The solid line corresponds to the orientation (in radians) of the instantaneous direction of translation of the centroid of the wrench, and the dashed line corresponds to the orientation (in radians) of the principal axis of the wrench.

and yaw, i.e. in locally planar, translational motion. Long sequences are needed to detect basic maneuvers such as turning or lane changing. In Section 6.2 we analyze two examples: an accelerating van (essentially linear motion) and a turning taxi.

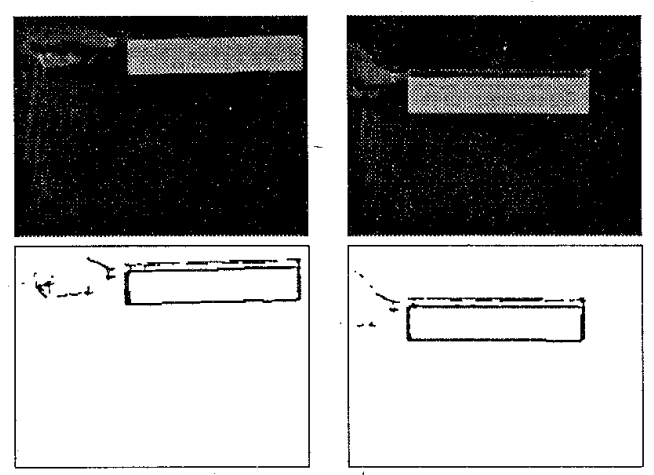

Figure 4: An experiment using a saw: frames 30 and 100. Top images: the input images. Bottom images: results of flow computation.

\subsection{Motions of Tools}

We tested our motion analysis algorithm under full perspective on two image sequences of tools in motion. The first sequence, shown in Figure 1, was a 200-image sequence of the movement of a wrench tightening a bolt.

The motion of the wrench was a rotation (to turn the bolt) around an axis approximately orthogonal to the plane of the image. $:$ The rotational velocity is shown in Figure 2 ; it is given in radians/sec and it corresponds to the scaled curvature $v \kappa$. Figure 3 shows

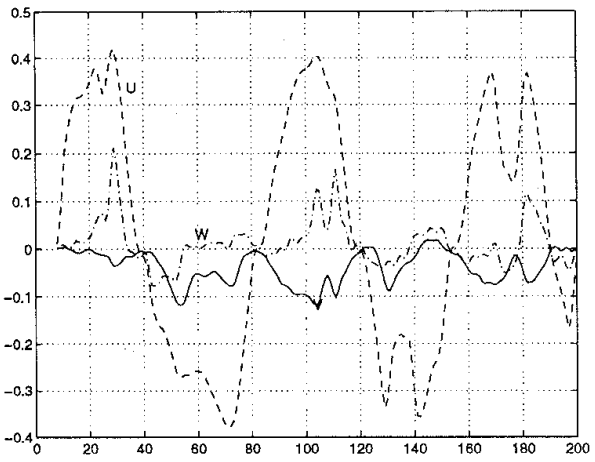

Figure 5: Results of experiments on the saw sequence. $U, V, W$ are the scaled (by an unknown distance $Z_{0}^{-1}$ ) components of the relative translational velocity.

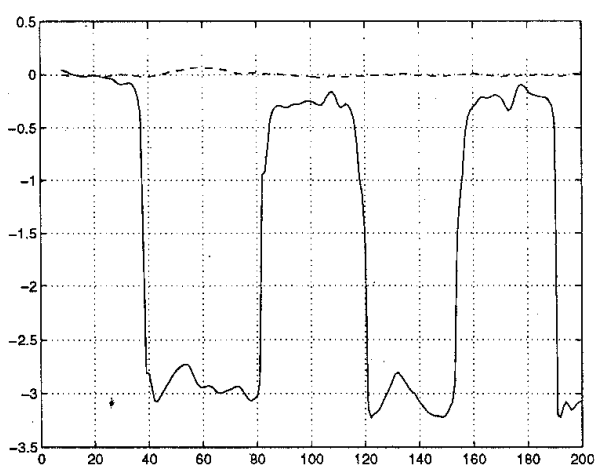

Figure 6: Results of experiments on the saw sequence. The solid line corresponds to the orientation (in radians) of the instantaneous direction of motion of the saw, and the dashed line corresponds to the orientation (in radians) of the principal axis of the saw.

the orientation of the principal axis of the wrench and the instantaneous translational velocity vector of its centroid (obtained using (13)), both measured in radians. As we see, the translational velocity vector remains approximately orthogonal to the principal axis throughout the motion sequence. The Frenet-Serret frame has its binormal $\vec{b}$ in the direction of the negative $z$-axis, its tangent $\overrightarrow{\mathbf{t}}$ in the image plane and orthogonal to the principal axis of the wrench, and its normal $\overrightarrow{\mathbf{n}}$ in the image plane and oriented from the centroid of the wrench toward the bolt.

We also tested our motion analysis algorithm on a 200 -image sequence of a saw doing a periodic motion. Figure 4 presents part of the sequence. Flow results are given below each image. The motion of the saw was pure translation $(\|\vec{\omega}\|=0)$. As can be seen from Figure 5 the motion is mostly fronto-parallel (the $z$ component of the translational velocity is small). The motion is periodic in the direction of the principal axis of inertia. It is a simple case of a (periodic) straight line motion with the Frenet-Serret frame correspond- 

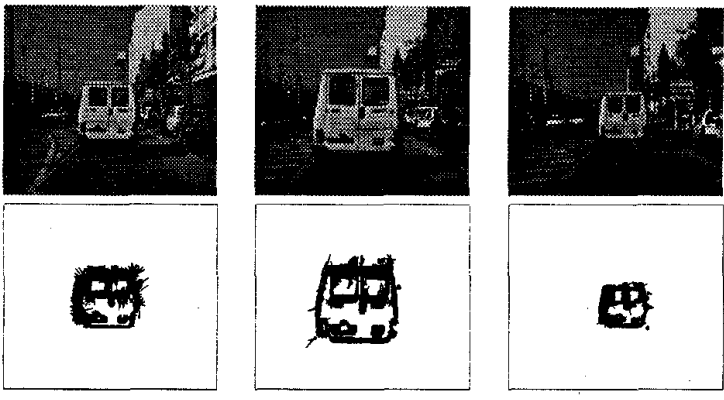

Figure 7: Frames 5, 25, and 45 of the van sequence. The normal flow results are shown below the corresponding image frames.

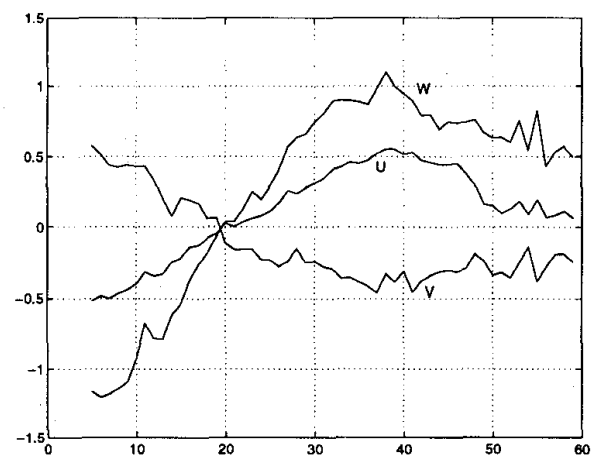

Figure 8: Results of experiments on the van sequence. $U, V, W$ are the scaled (by an unknown distance $Z_{c}^{-1}$ ) components of the relative translational velocity .

ing to the principal axes of the saw; $\overrightarrow{\mathbf{t}}$ corresponds to the longest axis, and $\vec{b}$ to the shortest axis.

These graphs show that the motion components have a simple behavior; before they reach their extremal values they can be approximated by straight lines, indicating constant relative accelerations.

\subsection{Motions of Vehicles}

Here we also used two image sequences, and we used the algorithms for weak perspective. In the first experiment we used an image sequence of a van taken from another vehicle following the van. The sequence consisted of 56 frames (slightly less than two seconds). Figure 7 shows frames $5,15,25$, and 35 as well as the corresponding normal flow on the van. Figure 8 shows estimated values of $U Z_{c}^{-1}, V Z_{c}^{-1}$, and $W Z_{c}^{-1}$. These values correspond to the relative translation of the van and the vehicle carrying the camera (observer coordinate system). Because of our choice of the coordinate system the rate of approach $\nu$ corresponds to the negative of $W Z_{c}^{-1}$, i.e. $\nu=-W Z_{c}^{-1}$. The graph shows

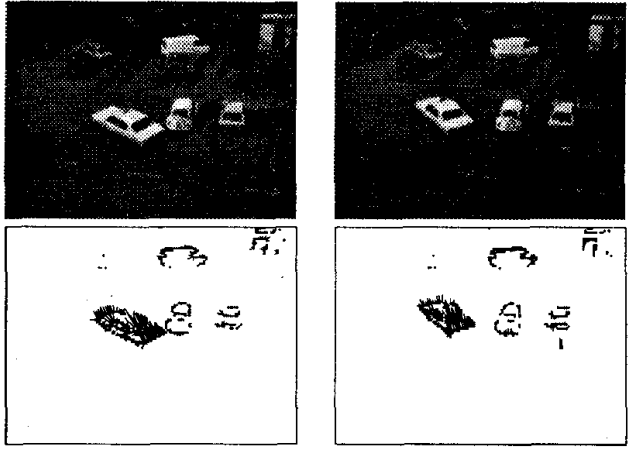

Figure 9: A taxi sequence: frames 1 and 21. Top images: the input images. Bottom images: results of flow computation.

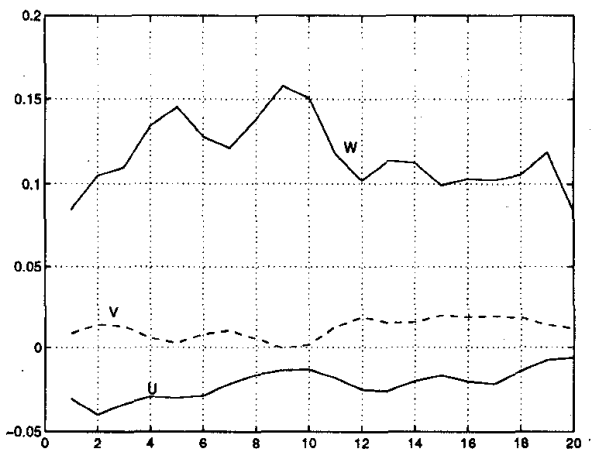

Figure 10: Results of experiments on the taxi sequence. $U, V, W$ are the scaled (by an unknown distance $Z_{c}^{-1}$ ) components of the relative translational velocity .

that there is an impending collision (rate of approach greater than $\left.1 \mathrm{sec}^{-1}\right)$. Around the 20th frame the rate of approach becomes zero (as do all the velocity components) and after that it becomes negative because the van starts pulling away from the vehicle carrying the camera.

In the second experiment we used an image sequence of a turning taxi taken by a stationary camera. The sequence consisted of 21 frames. Figure 9 shows frames $1,9,15$ and 21 as well as the corresponding normal flow on the vehicles. Figure 10 shows estimated values of $U Z_{c}^{-1}, V Z_{c}^{-1}$, and $W Z_{c}^{-1}$. These values correspond to the relative translation of the taxi. The graph shows that there is a large $W$ component in the turn (the taxi is receding), and that the turn is to the right (negative $U$, positive $V$ ). 


\section{Conclusions}

Many types of common objects, such as tools and vehicles, usually move in simple ways when they are wielded or driven: The natural axes of the object tend to remain aligned with the local trihedron defined by the object's trajectory. In this paper we have considered the relationship between this constrained motion and the object's geometry. To analyze this relationship we have used two frames: the object frame and the frame of the motion trajectory. Assuming a constant relationship between the object frame and the motion frame during the motion, we have used Frenet-Serret motion as a motion model. Using the Frenet-Serret frame has provided us with an ability to understand motion over a long time period.

We have derived equations for understanding the motions of tools and vehicles under full and weak perspective. We have recovered descriptions of an object's motion and the space curve along which the object moves, using relatively long image sequences. The motion and trajectory parameters provide a lowlevel description for understanding the motions of vehicles. For understanding tools in motion one needs additional knowledge about the tool and the context. This is a direction for further research.

It is the need for efficient force transfer that imposes a simple and constant relationship between the natural axes of the object and the motion trajectory. We have used this functional constraint in analyzing the motions of tools and ground vehicles. Expanding this analysis to other classes of objects (e.g. air vehicles), as well as expanding the vocabulary that describes the behavior of tools and vehicles (sharp turn, skid, etc.) [16], are other directions for future research.

\section{Acknowledgments}

The authors would like to thank Professor G.W. Stewart for bringing to our attention the method described in [12].

\section{References}

[1] T. J. Broida and R. Chellappa. Estimation of object motion parameters from noisy images. IEEE Transactions on Pattern Analysis and Machine Intelligence, 8:90-99, 1986.

[2] A. M. Bruckstein, R. J. Holt, and A. N. Netravali. How to catch a crook. Journal of Visual Communication and Image Representation, 5:273-281, 1994.

[3] A. M. Bruckstein, R. J. Holt, and A. N. Netravali. How to track a flying saucer. Journal of Visual Communication and Image Representation, 7:196204, 1996.

[4] H. Buxton and R. Howarth. Watching behavior: The role of context and learning. In Proc. Inter- national Conference on Image Processing, pages B797-800, 1996.

[5] C. Cedras and M. Shah. Motion-based recognition: A survey. Image and Vision Computing, 13:129155,1995 .

[6] Z. Duric, J. Fayman, and E. Rivlin. Function from motion. IEEE Transactions on Pattern Analysis and Machine Intelligence, 15:105-122, 1995.

[7] Z. Duric and A. Rosenfeld. Image sequence stabilization in real time. Real-Time Imaging, 2:271284, 1996.

[8] Z. Duric, A. Rosenfeld, and L. S. Davis. Egomotion analysis based on the Frenet-Serret motion model. International Journal of Computer Vision, $15: 105-122,1995$.

[9] Z. Duric, E. Rivlin, and A. Rosenfeld. Understanding object motion. Technical Report CAR-TR-854, University of Maryland, College Park, MD, 1997.

[10] Z. Duric, E. Rivlin, and A. Rosenfeld. Understanding object motion. In Proc. DARPA Image Understanding Workshop, pages 143-152, May. 1997.

[11] S. Engel and J. Rubin. Detecting visual motion boundaries. In Proc. Workshop on Motion, pages 107-111, 1986.

[12] G.H. Golub and V. Pereyra. The differentiation of pseudo-inverses and nonlinear least squares problems whose variables separate. SIAM Journal on Numerical Analysis, 10:413-432, 1973.

[13] K. Gould and M. Shah. The trajectory primal sketch: A multi-scale scheme for representing motion characteristics. In Proc. Conference on Computer Vision and Pattern Recognition, pages $79-$ 85, 1989.

[14] N. Johnson and D. Hogg. Learning the distribution of object trajectories for event recognition. Image and Vision Computing, 14:609-615, 1996.

[15] D. Koller, K. Daniilidis, and H.H. Nagel. Modelbased object tracking in monocular image sequences of road traffic scenes. International Journal of Computer Vision, 10:257-282, 1993.

[16] D. Koller, H. Heinze, and H.H. Nagel. Algorithmic characterization of vehicle trajectories from image sequences by motion verbs. In Proc. Conference on Computer Vision and Pattern Recognition, pages 90-95, 1991.

[17] E. Kreyszig. Differential Geometry. University of Toronto Press, Toronto, Canada, 1959.

[18] J. Weng, T.S. Huang, and N. Ahuja. 3-D motion estimation, understanding, and prediction from noisy image sequences. IEEE Transactions on Pattern Analysis and Machine Intelligence, 9:370-389, 1987. 